\title{
Orientation discrimination across the visual field: Size estimates near contrast threshold
}

\author{
SHARON L. SALLY \\ University of Rochester, Rochester, New York \\ FRÉDÉRIC J. A. M. POIRIER \\ York University, Toronto, Ontario, Canada \\ and \\ RICK GURNSEY \\ Concordia University, Montreal, Quebec, Canada
}

\begin{abstract}
Performance in detection and discrimination tasks can often be made equal across the visual field through appropriate stimulus scaling. The parameter $E_{2}$ is used to characterize the rate at which stimulus dimensions (e.g., size or contrast) must increase in order to achieve foveal levels of performance. We calculated both size and contrast $E_{2}$ values for orientation discrimination using a spatial scaling procedure that involves measuring combination size and contrast thresholds for stimuli with constant sizeto-contrast ratios. $E_{2}$ values for size scaling were $5.77^{\circ}$ and $5.92^{\circ}$. These values are three to four times larger than those recovered previously using similar stimuli at contrasts well above detection threshold (Sally \& Gurnsey, 2003). $E_{2}$ values for contrast scaling were $324.2^{\circ}$ and $44.3^{\circ}$, indicating that for large stimuli little contrast scaling (.3\% to $2.3 \%$ increase) was required in order to equate performance in the fovea and the largest eccentricity $\left(10^{\circ}\right)$. A similar pattern of results was found using a spatial scaling method that involves measuring contrast thresholds for target identification as a function of size across eccentricities. We conclude that the size scaling for orientation discrimination at near-threshold stimulus contrasts is much larger than that required at suprathreshold contrasts. This may arise, at least in part, from contrast-dependent changes in mechanisms that subserve task performance.
\end{abstract}

Performance in many spatial vision tasks declines when a stimulus of a constant size is presented at greater retinal eccentricities (e.g., Rovamo \& Virsu, 1979; Rovamo, Virsu, \& Näsänen, 1978; Weymouth, 1958). However, performance can often be made equal across the visual field by magnifying peripherally presented stimuli in all dimensions according to

$$
F=1+\frac{E}{E_{2}},
$$

where $E_{2}$ indicates that eccentricity $(E)$ in degrees at which the size of a stimulus must be doubled relative to the foveal standard to achieve equivalent performance (Levi, Klein, \& Aitsebaomo, 1984, 1985).

The idea that performance can be equated across the visual field through an appropriate scaling factor was originally proposed by Rovamo et al. (1978) and Koenderink, Bouman, Bueno de Mesquita, and Slappendel (1978). In these studies, stimuli were scaled in inverse proportion to the size of the proposed cortical neural pro-

This research was supported by NSERC and FCAR research grants to R.G. Portions of this article were presented at the 2002 annual meeting of the Vision Sciences Society, Sarasota, Florida. Correspondence should be addressed to R. Gurnsey, Concordia University, Department of Psychology, 7141 Sherbrooke W., Montreal, QC, H4B 1R6 Canada (e-mail: rick.gurnsey@concordia.ca). jection area, a procedure known as $M$-scaling. It was assumed that performance for all tasks could be made equal across eccentricities by the use of a single set of predetermined scaling factors, one for each principal meridian of the visual field. Later research, however, suggested that the amount of peripheral size scaling required to equate task performance depends on task demands (Klein \& Levi, 1987; Levi et al., 1985; Westheimer, 1982). To overcome limitations associated with M-scaling, a procedure known as spatial scaling or S-scaling, was introduced (Johnston, 1987; Johnston \& Wright, 1986; Saarinen, Rovamo, \& Virsu, 1989; Watson, 1987; Wright, 1987). The technique makes no a priori assumptions concerning the size of peripheral magnification factors. Task performance (e.g., proportion correct responses or discrimination thresholds) is measured for a set of stimulus sizes at each eccentricity and is then plotted as a function of stimulus size. If size scaling alone is sufficient to overcome the peripheral sensitivity losses, performance-versus-size curves at all eccentricities will have a similar shape on logarithmic axes and will be laterally shifted versions of each other. The amount by which the peripheral curves must be shifted on the size axis in order to superimpose all data determines size scaling.

Implicit in spatial scaling theory is the assumption that achieving optimal performance at each eccentricity requires an appropriate match between stimulus dimen- 
sions and the mechanisms they engage. The major limitation on peripheral performance is assumed to be an eccentricity-dependent variation in the spatial scale of the underlying neural mechanisms. According to Watson (1987), this amounts to assuming that visual processing is homogeneous throughout the visual field apart from a change in the scale of the local mechanisms at each peripheral location; the parameter $E_{2}$ is thought to reflect this eccentricity-dependent scale change.

Mäkelä, Näsänen, Rovamo, and Melmoth (2001) argued that size-scaling estimates may be erroneous if one does not explicitly consider the role of stimulus contrast. They had subjects perform a face-discrimination task and measured the contrast required to perform the task as a function of stimulus size at a range of eccentricities. The identification sensitivity versus size curves at each eccentricity reached different asymptotic levels, indicating that a shift along the size axis was insufficient to bring all curves into alignment. The curves had to be shifted along the contrast axis as well as the size axis to superimpose all the data. When identification sensitivity curves were aligned with a single lateral shift, relatively large shifts were required, the resulting values of $E_{2 s i z e}$ were small $\left(1.43^{\circ}\right.$ and $\left.1.87^{\circ}\right)$, leaving substantial variability in the data unexplained. When data were shifted along the size and contrast axes (i.e., double scaling), smaller lateral shifts were required to align the functions, and hence the $E_{2 \text { size }}$ values recovered were substantially larger $\left(2.73^{\circ}\right.$ and $\left.3.19^{\circ}\right)$.

In a related study, Sally and Gurnsey (2003) concluded that a failure to control for perceptual contrast may influence recovered $E_{2 \text { size }}$ values in an orientationdiscrimination task. However, Sally and Gurnsey (2003) concluded that failure to control perceptual contrast tends to inflate $E_{2 \text { size }}$ values. Using a conventional orientationdiscrimination task in which orientation thresholds were measured for a range of stimulus sizes and eccentricities, Sally and Gurnsey (2003) reported an average $E_{2 \text { size }}$ value of $2.36^{\circ}$ for broadband stimuli (identical to the stimuli used in the experiments reported below). They proposed that as stimulus size is reduced, a concomitant reduction in perceptual contrast would have effects similar to reductions in physical stimulus contrast (e.g., increased orientation thresholds). Thus, at small stimulus sizes both perceptual contrast and orientation sensitivity combine to determine orientation-discrimination thresholds. An examination of a number of previous reports suggested that the inclusion of orientation thresholds obtained with very small stimuli in the calculation of $E_{2 \text { size }}$ values tends to inflate the recovered $E_{2 s i z e}$ values. That is, when reduced perceptual contrast inflates orientation-discrimination thresholds, $E_{2 \text { size }}$ values are also inflated. To eliminate the influence of perceptual contrast, Sally and Gurnsey (2003) presented stimuli at physical contrasts that were high enough for orientation thresholds to reach an asymptotic level. The average $E_{2 \text { size }}$ values were reduced $42 \%$ from $2.36^{\circ}$ to $1.38^{\circ}$.

Two questions arise from the contrasting results of Sally and Gurnsey (2003), in which it was concluded that controlling contrast leads to smaller $E_{2 \text { size }}$ values, and those of Mäkelä et al. (2001), in which it was concluded that controlling contrast leads to larger $E_{2 \text { size }}$ values. The first and most significant question is whether $E_{2 \text { size }}$ values recovered at near-threshold contrasts are, in fact, larger than those recovered at contrasts far above detection threshold. The literature suggests that this is generally the case. For example, $E_{2}$ values obtained at nearthreshold contrasts for face discrimination (Mäkelä et al., 2001) and detection of phase distortions (Melmoth, Kukkonen, Mäkelä, \& Rovamo, 2000b) are much larger than those obtained at contrasts well above detection threshold for orientation discrimination (Mäkelä, Whitaker, \& Rovamo, 1993; Sally \& Gurnsey, 2003), vernier acuity (Whitaker, Rovamo, MacVeigh, \& Mäkelä, 1992), and curvature detection and discrimination (Whitaker, Latham, Mäkelä, \& Rovamo, 1993). Unfortunately, in none of this work are $E_{2 \text { size }}$ values compared using the same stimuli at contrast near to and well above detection threshold. As mentioned earlier, Sally and Gurnsey (2003) examined orientation discrimination across the visual field using stimuli well above detection threshold. Here, we use exactly the same stimuli but assess orientation-discrimination performance at contrasts near detection threshold.

Emerging psychophysical and physiological evidence suggests that the spatial structure of the receptive fields of cortical mechanisms supporting orientation discrimination is contrast dependent (Kapadia, Westheimer, \& Gilbert, 1999; Mareschal, Henrie, \& Shapley, 2002; Mareschal \& Shapley, 2004; Sceniak, Hawken, \& Shapley, 2002; Sceniak, Ringach, Hawken, \& Shapley, 1999). For example, Kapadia et al. (1999) showed that length summation in V1 cells increased with decreases in contrast. It is clearly possible that changes in receptive field structure with changes in contrast may contribute to the contrast dependence of $E_{2 \text { size }}$ values discussed above. Therefore, it is natural to address this question within the context of the orientation-discrimination task used here, because size and orientation selectivity are prominent features of the cortical mechanisms whose receptive fields show contrast-dependent reorganization.

A second question that arises from the results of Sally and Gurnsey (2003) and those of Mäkelä et al. (2001) is whether both size scaling and contrast scaling are required in an orientation-discrimination task conducted at near-threshold contrasts. Melmoth et al. (2000b) argued that contrast scaling is generally not required at contrasts well above detection threshold, even for "complex" tasks such as letter identification (i.e., Farrell \& Desmarais, 1990; Higgins, Arditi, \& Knoblauch, 1996). Contrast scaling may only be required for complex tasks conducted at near-detection-threshold levels of stimulus contrast. ${ }^{1}$ For example, both size and contrast scaling are required for alphanumeric character recognition (Strasburger, Harvey, \& Rentschler, 1991; Strasburger, Rentschler, \& Harvey, 1994) and face discrimination (Mäkelä et al., 2001; Melmoth, Kukkonen, Mäkelä, \& Rovamo, 2000a). The "simple" task of detecting phase distortions can be equated across eccentricities through size scaling alone (Mel- 
moth et al., 2000b). If simple tasks can be associated with limitations imposed by the primary visual cortex and earlier, orientation discrimination should not require contrast scaling.

We measure size and contrast $E_{2}$ values using an adaptation of the method introduced by Poirier and Gurnsey (2002). This procedure (henceforth the Poirier-Gurnsey procedure) involves measuring scale-thresholds for stimuli having a fixed ratio of size to contrast; each such ratio is referred to as a configuration. Each configuration defines a straight line emanating from the origin of a space of sizes and contrasts; a scale threshold, therefore, represents a combination of stimulus size and stimulus contrast that elicits a fixed level of performance. $E_{2 \text { size }}$ and $E_{2 \text { contrast }}$ values are recovered by determining the horizontal and vertical shifts required to align the data. $^{2}$

Experiments were also conducted using the method reported by Melmoth et al. (2000a, 2000b) (henceforth the Melmoth procedure). The Melmoth procedure involves measuring contrast thresholds for fixed stimulus sizes at various eccentricities. In essence, contrast sensitivity functions (CSFs) are measured at a range of eccentricities, and $E_{2 \text { size }}$ and $E_{2 \text { contrast }}$ values are recovered by determining the horizontal and vertical shifts required to align these CSFs. Thus, both procedures involve measuring orientation-discrimination performance at contrasts near detection threshold. Overall, the two procedures yielded very similar results, so those obtained using the Melmoth procedure are reported fairly briefly to reduce the presentation of redundant information.

To summarize, we address three questions in the present article. First, are $E_{2 \text { size }}$ values recovered at nearthreshold contrasts greater than those recovered at contrasts well above detection threshold? Second, is contrast scaling required in the orientation-discrimination task? If so, does the need to scale contrast have a substantial effect on the $E_{2 \text { size }}$ values recovered? And third, do the PoirierGurnsey and Melmoth methods yield similar results and lead to similar conclusions about the above questions?

\section{METHOD}

\section{Subjects}

Two subjects, including one of the authors (S.S.), participated in the experiment. S.S. and S.M. were fully corrected myopes, and both wore their respective distance correction during testing.

\section{Apparatus}

Stimulus images were generated using a Power Mac G4 computer and presented on a 21-in. Sony Trinitron CRT color monitor having pixel resolution of $1,600 \times 1,200$. Pixel width was $0.233 \mathrm{~mm}$, and the frame refresh rate was $85 \mathrm{~Hz}$. The background luminance of displays was $13.0 \mathrm{~cd} / \mathrm{m}^{2}$. Luminance resolution was increased by combining color channels with a video summation device (Pelli \& Zhang, 1991), allowing contrast resolution of up to 12-bit accuracy. Software from the VideoToolbox (Pelli, 1997) was used to calibrate the device and correct for display nonlinearities. Absolute luminance levels were determined with a Minolta CS-100 photometer.

\section{Stimuli}

The stimuli were created and experiments were run in the Matlab (Mathworks Ltd.) programming environment using Psychtoolbox code (Brainard, 1997). The stimuli were line patterns having Gaussian cross sections along their minor axes. One of the line patterns was vertical, and the other was tilted $1.5^{\circ}$ from vertical. The width of each line $( \pm 2 \sigma)$ was $11 \%$ of its length. These stimuli were identical to those used to measure orientation thresholds at high, suprathreshold levels of stimulus contrast (Sally \& Gurnsey, 2003). The fixed orientation difference of $1.5^{\circ}$ was selected because we had determined in pilot testing that this was approximately the smallest angular difference at which correct discriminations could be made for a reasonable range of stimulus sizes and eccentricities. We note that the average minimum discriminable orientation difference at high contrasts was $0.56^{\circ}$ and $0.55^{\circ}$ for SS and SM, respectively (Sally \& Gurnsey, 2003).

\section{Procedure}

The following procedures were employed with both data-sampling methods. The stimuli were presented at $0^{\circ}, 2.5^{\circ}, 5^{\circ}$, and $10^{\circ}$ eccentricity along the horizontal meridian in the right visual field. Eccentricity was defined as distance from fixation to the center of the stimulus. A fixation spot (six pixels) was presented for all eccentricities except $0^{\circ}$, and auditory feedback was provided after each response. The experiment was conducted in a dimly lit room, and viewing was binocular.

A trial consisted of the sequential presentation of two line stimuli; one was vertical and the other was tilted. Each was presented for $200 \mathrm{msec}$ separated by an interstimulus interval of $300 \mathrm{msec}$. The subject reported via the mouse which interval contained the tilted stimulus - that is, a two-interval forced choice (2IFC). Stimulus location was jittered from trial to trial by $5 \%$ of its height so that absolute stimulus location could not provide an orientation cue.

Thresholds were obtained using an adaptive procedure (QUEST; Pelli, 1987; Watson \& Pelli, 1983) that assumes an underlying Weibull function. The $82 \%$ correct detection level was taken as threshold. Each threshold estimate resulted from approximately 75 trials, and the final threshold represents a mean of two to four estimates.

\section{The Poirier-Gurnsey Procedure}

Data-collection method. The left panel of Figure 1 summarizes the underpinnings of the Poirier-Gurnsey procedure. The curved lines represent combinations of stimulus size and stimulus contrast that elicit threshold-level performance. The solid line represents foveal data, and the broken line represents $10^{\circ}$ in the periphery. A notable feature of these curves is that they become asymptotic with respect to the size and contrast axes. For the present stimuli, this means that at each eccentricity thresholds are determined by an asymptotically low contrast for large stimuli. And for very high contrasts, there is an asymptotic limit to stimulus size. The diagonal lines depict combinations of size and contrast having a constant ratio. We refer to these combinations as having the same configuration (Poirier \& Gurnsey, 2002). In a linear space, these configuration lines emanate from the origin. The stimuli are said to increase in scale as one moves from the origin outward. We determined scale thresholds for a number of configurations at each eccentricity. Computing thresholds in this way may be referred to as sampling along configuration lines. The terms data-collection method and sampling method are used interchangeably.

The initial set of configurations was determined in pilot experiments by measuring the minimum size at which stimulus identification could be made across eccentricities when contrast was maximal. The maximum size was chosen such that stimulus size at contrast threshold would not exceed the limits of the display. Con- 

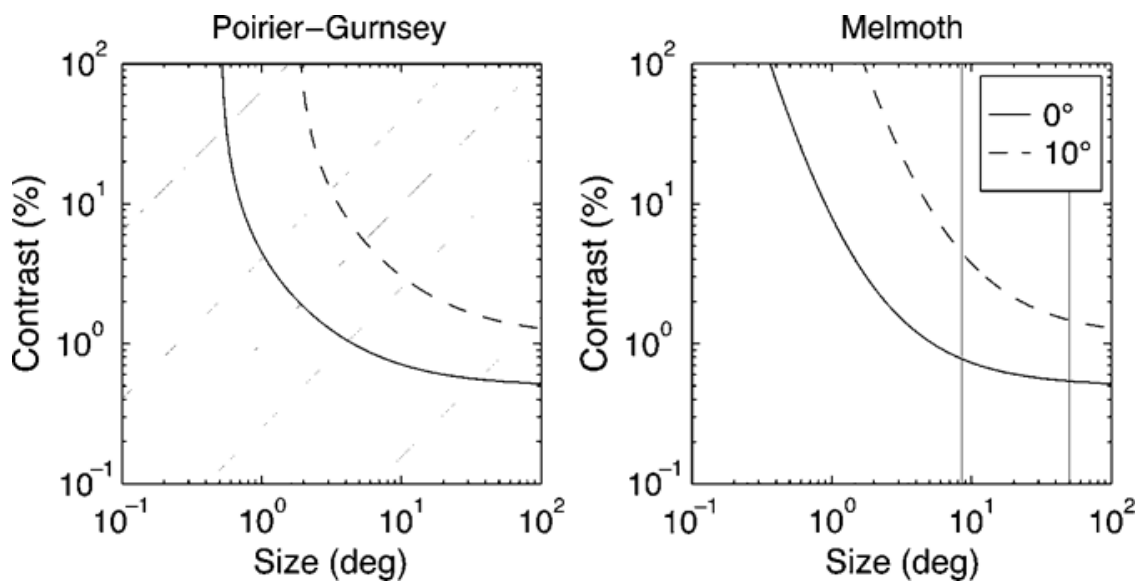

Figure 1. Illustration of the conceptual frameworks of Poirier and Gurnsey (2002) and Melmoth, Kukkonen, Mäkelä, and Rovamo (2000a, 2000b). In the Poirier-Gurnsey sampling method, stimuli vary simultaneously in size and in contrast, in such a way that size is proportional to contrast. Each diagonal line represents a different configuration - that is, ratio of size to contrast. Stimulus scale increases as one moves out along a configuration line, and threshold represents the scale that elicits $82 \%$ correct responses. In the Melmoth sampling method, contrast thresholds are determined for a range of stimulus sizes at several eccentricities.

figuration lines were then selected to be at equal logarithmic steps within this space. For each configuration, line length was a fixed multiple of stimulus contrast; for example, a contrast of .1 and a multiplier of 64 corresponds to a line that is $6.4^{\circ}$ in length. Nine different configurations were tested at all eccentricities. These configurations were defined by line lengths that were 5.1, 10.2, 20.4, $40.8,81.6,163.2,326.4,652.9$, and $1,305.8 \times$ stimulus contrast. A configuration of 2.55 was tested at eccentricities of $0^{\circ}, 2.5^{\circ}$, and $5^{\circ}$, and a configuration of 1.28 was tested at $0^{\circ}$. A viewing distance of $100 \mathrm{~cm}$ was used for all but the smallest and largest multipliers. The viewing distance was decreased to $50 \mathrm{~cm}$ for the largest multipliers $\left(652.9^{\circ}\right.$ and $\left.1,305.8^{\circ}\right)$. The maximum stimulus presentation size was set at $24^{\circ}$ for these two conditions, and all thresholds were below this limit. Viewing distance was increased to $200 \mathrm{~cm}$ for multipliers that yielded the smallest stimulus sizes on the screen. All eccentricities were tested for one multiplier (configuration) before moving to the next. The configurations were tested in a random order.

Poirier and Gurnsey (2002) proposed that scale thresholds at each eccentricity conform to a rectangular parabola (Serway, 1992; Strasburger et al., 1994) that has the form

$$
\left(s-s_{\min }\right)\left(c-c_{\min }\right)=\Omega^{2},
$$

where $s$ and $c$ represent combinations of size and contrast at orientation-discrimination threshold. $s_{\min }$ and $c_{\min }$ are size and contrast limits such that thresholds have reached a plateau or saturation level with respect to these variables. $\Omega^{2}$ determines the curvature of the function at intermediate values of $s$ and $c$; the curves in the left panel of Figure 1 are defined by Equation 2 .

Data-fitting method. Size scaling theory holds that data at all eccentricities should collapse onto the same function by scaling the sizes $(x)$ of all stimuli at each eccentricity $(E)$ by an appropriate factor:

$$
x_{\text {scaled }}=\frac{x_{e}}{F},
$$

where $F_{\text {size }}=1+E / E_{2 \text { size }}$, as given in Equation 1. Contrast can be scaled in an identical manner.

Shifting the peripheral curves down to the left in log-log space corresponds to dividing the parameters ( $s$ and $c$ ) of the rectangular parabola by linear scaling factors associated with size and contrast. The scaling for parameter $\Omega^{2}$ is equal to the product of $F_{\text {size }}$ and
$F_{\text {contrast }}$ (see Poirier \& Gurnsey, 2002). The entire data set was scaled by finding parameters for $s_{\min }, c_{\min }, \Omega^{2}, E_{2 \text { size }}$ and $E_{2 \text { contrast }}$ that minimize the squared deviation of the data from the parametric curve along the configuration lines. In fact, the fitting was done in a space that is a $45^{\circ}$ rotation of that shown in the left panel of Figure 1. In this space, configuration becomes the independent variable ( $x$ axis), and scale becomes the dependent variable ( $y$ axis). We minimized the sum of squared deviations of the data from the best-fitting functions in this rotated space so that $r^{2}$ could be used to express the proportion of variability in the data explained by the fit. [For further details, see Poirier and Gurnsey, 2002.]

The data were fit using the error-minimization routine provided in Matlab (Mathworks Ltd.); this routine (fmins) uses the NelderMead simplex (direct search) method. Numerical solutions found in this way may represent local rather than global minima. Therefore, we ran the minimization routine 20 times for each fit, each starting from a different randomly chosen initial condition, and we report the best fits (highest $r^{2}$ values) obtained in this way.

\section{The Melmoth Procedure}

Data-collection method. The contrast thresholds for identification of the target stimulus were obtained for a range of logarithmically spaced stimulus sizes at each eccentricity. Retinal sizes of $0.375^{\circ}-24^{\circ}$ were achieved by varying the viewing distance and/or changing the stimulus dimensions on the screen. Stimulus sizes of $3^{\circ}, 1.5^{\circ}, 0.75^{\circ}$, and $0.375^{\circ}$ were obtained by fixing stimulus size on the screen and varying the viewing distance from 50 to $400 \mathrm{~cm}$. Stimulus sizes of $6^{\circ}, 12^{\circ}, 18^{\circ}$, and $24^{\circ}$ were generated at a viewing distance of $50 \mathrm{~cm}$ by increasing stimulus dimensions from 112 to 448 pixels. Pixel resolution was also changed to $800 \times 600$ to create the three largest sizes $\left(12^{\circ}, 18^{\circ}\right.$, and $\left.24^{\circ}\right)$. All eccentricities were tested for one stimulus size before moving to the next. The stimulus sizes were tested in a random order.

Melmoth et al. (2000a, 2000b) and Mäkelä et al. (2001) have assumed that CSFs at all eccentricities can be described by the function

$$
S=S_{\max }\left[1+\left(\frac{S_{\text {crit }}}{x}\right) p\right]^{-n},
$$

where $S$ is identification sensitivity, $S_{\max }$ is the maximum contrast sensitivity, $S_{\text {crit }}$ refers to the critical image size marking the transi- 
tion between the steeply ascending and saturating parts of Equation 4 , and $x$ is line length (size). The product of the exponents $(p n)$ indicates the slope of the increasing part of the function. We use the reciprocal of Equation 4 to facilitate comparison with the Poirier-Gurnsey method. The right panel of Figure 1 summarizes underpinnings of the method employed by Melmoth et al. (2000a, $2000 \mathrm{~b}$ ). The curved lines represent hypothetical contrast thresholds for a range of stimulus sizes (indicated by the vertical lines) at two eccentricities (the solid line represents foveal data, and the broken line represents $10^{\circ}$ in the periphery). Collecting contrast thresholds may be described as sampling along lines that are parallel to the contrast axis.

Data-fitting method. The entire data set for each subject was size- and contrast-scaled by finding parameters for $S_{\text {crit }}, S_{\max }, E_{2 \text { con- }}$ trast $, E_{2 \text { size }}, p$, and $n$ that minimize the deviation of the data from the parametric curve. Following Melmoth et al. (2000a, 2000b), the measure of deviation used was RMS error, defined as

$$
e_{\mathrm{rms}}=\sqrt{\frac{1}{n} \sum_{i}^{n}\left(\log Y_{i(\mathrm{est})}-\log Y_{i}\right)^{2}},
$$

where $n$ is the number of data points, $Y_{i}$ is a measured data point, and $Y_{i(\text { est })}$ is the value predicted by the parametric function. Goodness of the fit is expressed as $G=1-e_{\mathrm{rms}}$. The data set was also scaled by finding the parameters that minimize the squared devia- tion $\left(r^{2}\right)$ of the data from the parametric curve. These two measures yielded virtually identical $E_{2}$ values. We therefore report results obtained using the latter method, because it is the measure of deviation used in the Poirier-Gurnsey method, and we also present $G$ values for all fits. The error-minimization routine was as described earlier. We report the best fits (highest $r^{2}$ and $G$ values) obtained from 20 runs with different initial conditions.

\section{RESULTS}

Figure 2 (top panels) shows combinations of stimulus size and contrast that elicit threshold level performance for data obtained using the Poirier-Gurnsey procedure. Also shown are the best-fitting parametric functions. Note that the data have the same general form at all eccentricities, and minimum Michelson contrasts are reasonably similar for the 2 subjects $(.0105$ and .0084 for S.S. and S.M., respectively). The scaled length data for the two subjects are shown in Figure 2 (bottom panels). The $r^{2}$ values were .93 and .94 for S.S. and S.M., respectively ( $G=.93$ for both subjects). We note that when residual error is assessed by the vertical distance
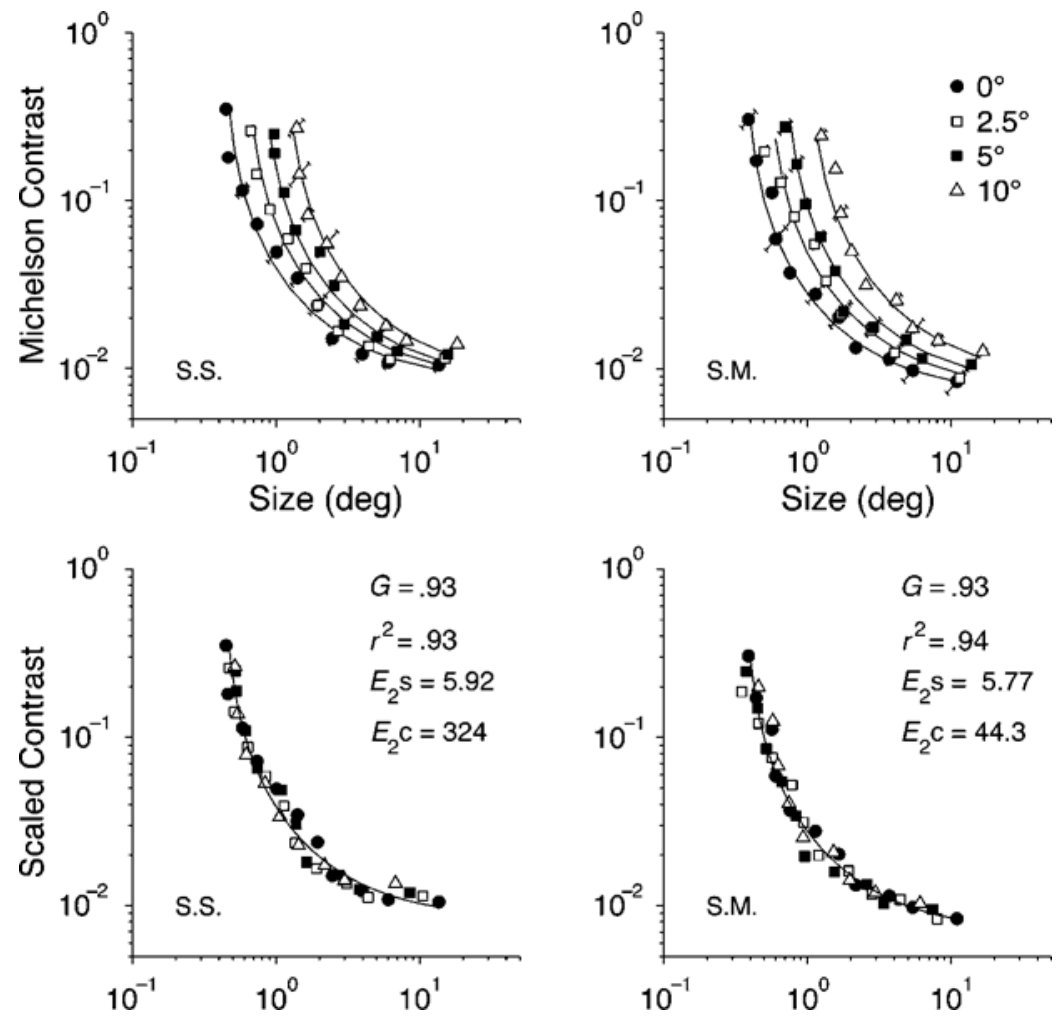

Figure 2. Data obtained for Subjects S.S. and S.M. using the Poirier-Gurnsey sampling method. Combinations of size (line length) and contrast at identification threshold are shown in the top panels. Contrast is plotted against line length, and standard errors are shown for each point. Eccentricities from $0^{\circ}-10^{\circ}: 0^{\circ}$ (filled circles), $2.5^{\circ}$ (unfilled squares), $5^{\circ}$ (filled squares), and $10^{\circ}$ (unfilled triangles) are shown in the top panels. Scaled line length and contrast data are shown in the bottom panels. $E_{2}$ values and fits $\left(G\right.$ and $\left.r^{2}\right)$ are as shown. Values for the fitting parameters $s_{\text {min }}, c_{\text {min }}$ and $\Omega^{2}$ were 0.42 , 0.0085 , and 0.1323 for S.S. and $0.3557,0.0071$, and 0.1153 for S.M., respectively. 
from the parametric curve (as in the Melmoth fitting method) the $r^{2}$ s obtained using the Poirier-Gurnsey fitting method increase to 99 .

The average $E_{2 \text { size }}$ obtained using the Poirier-Gurnsey data collection and fitting method was $5.85^{\circ}\left(5.92^{\circ}\right.$ and $5.77^{\circ}$ for S.S. and S.M., respectively). This value is 4.24 times greater than the average $E_{2 \text { size }}$ recovered using the same broadband stimuli at asymptotically high contrasts (1.38 ; Sally \& Gurnsey, 2003, Experiment 3). A onetailed paired $t$ test revealed that this difference is statistically significant $[t(1)=-27.06, p=.012]$. Furthermore, we refit the data of the present experiment, forcing the $E_{2 \text { size }}$ value to be 1.38 , while allowing all other parameters to vary freely. The explained variance dropped drastically for both subjects (from $93 \%$ to $69 \%$ for S.S. and from $94 \%$ to $75 \%$ for S.M.). Clearly, the $E_{2 \text { size }}$ recovered at asymptotically high contrasts does a poor job of size scaling the present data.

The average $E_{2 \text { contrast }}$ was $184.24^{\circ}\left(324.16^{\circ}\right.$ and $44.33^{\circ}$ for S.S. and S.M., respectively). $E_{2}$ values of this magnitude indicate that essentially no contrast scaling was required; for lines of sufficient length, contrast thresh- olds attain approximately the same minimal values. We refitted the data of the present experiment, forcing the $E_{2 \text { contrast }}$ value to be 20,000 (effectively infinity, meaning no scaling), while allowing all other parameters to vary freely. The explained variance remained essentially the same for both subjects (from $93 \%$ to $92.6 \%$ for S.S. and from $94 \%$ to $93.4 \%$ for S.M.).

Figure 3 (top panels) shows threshold Michelson contrast as a function of line length, obtained using the Melmoth sampling method along with the best-fitting parametric functions. Note that functions again have a similar form across eccentricities and that minimal thresholds are similar for the 2 subjects (.0096 and .0079 for S.S. and S.M., respectively), as well as across sampling methods. Figure 3 (bottom panels) shows the scaled data for the two subjects. $r^{2}$ values were again excellent; that is, .97 and .96 for S.S. and S.M., respectively $(G=.96$ and .95 , respectively).

One notable difference concerns the form of the threshold-versus-size curves recovered using the two sampling methods. These functions became parallel to the contrast axis when the Poirier-Gurnsey sampling
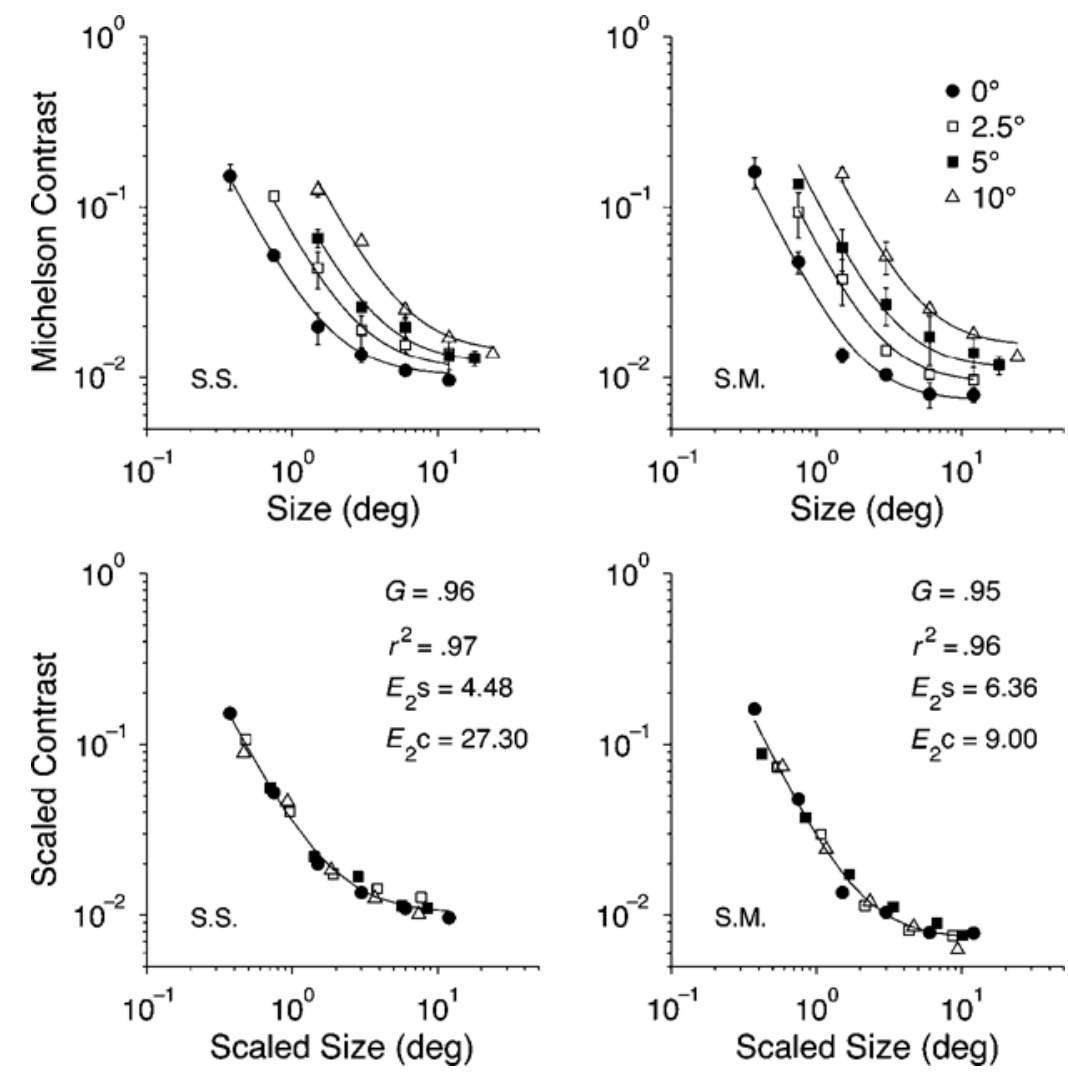

Figure 3. Data obtained for Subjects S.S. and S.M. using the Melmoth sampling method. Stimulus contrasts at identification threshold are shown in the panels. Contrast is plotted against line length, and standard errors are shown for each point. Symbols refer to eccentricities, as in Figure 2. Scaled line length and contrast data are shown in the bottom panels with $E_{2}$ values and fits $\left(G\right.$ and $\left.r^{2}\right)$. Values for the fitting parameters $S_{\max }, S_{\text {crit }}, p$, and $n$ were $97.77,1.75,1.76$, and 0.96 for S.S. and 137.35, 1.97, 1.95, and 0.90 for S.M., respectively. 
method was used, but not with the Melmoth sampling method. In other words, thresholds decline more gradually with increases in stimulus size when data are obtained with the Melmoth sampling method than with the Poirier-Gurnsey sampling method. We return to this point in the General Discussion section.

The average $E_{2 \text { size }}$ value obtained with the Melmoth data collection and fitting method was $5.42^{\circ}\left(4.48^{\circ}\right.$ and $6.36^{\circ}$ S.S. and S.M., respectively). These values compare with an average $E_{2 \text { size }}$ value of $5.85^{\circ}$ using the PoirierGurnsey sampling and fitting method. Thus, estimates of $E_{2 \text { size }}$ were very similar across procedures. The average $E_{2 \text { contrast }}$ value obtained with the Melmoth sampling method was $18.15^{\circ}\left(27.30^{\circ}\right.$ and $9.00^{\circ}$ for S.S. and S.M., respectively). ${ }^{3}$ The corresponding average $E_{2 \text { contrast }}$ value recovered using the Poirier-Gurnsey sampling method was $184.24^{\circ}$. Thus, there was some variation across procedures in the $E_{2 \text { contrast }}$ values.

Contrast thresholds for the largest stimulus sizes were somewhat lower at the furthest eccentricities when the Poirier-Gurnsey sampling method was used. This may have arisen either from the manner in which the size and contrast response space was sampled (see Figure 1) or the sampling density associated with the respective procedures. Overall, however, the $E_{2 \text { contrast }}$ values were very large, indicating that relatively little contrast scaling was required to equate performance across eccentricities.

Note that large differences in $E_{2}$ values become meaningless when the $E_{2}$ values in question are very large. The slope of Equation 1 [i.e., $F=1+E / E_{2}$ ] is $1 / E_{2}$. Thus, whether or not an $E_{2}$ value is $1.5^{\circ}$ or $5^{\circ}$ makes a big difference in the relative scaling required at fixation versus $10^{\circ}$ eccentricity, for example. If $E_{2}=1.5^{\circ}$, a stimulus at $10^{\circ}$ would have to be 6.67 times larger than the corresponding stimulus at fixation. If $E_{2}=5$, a stimulus at $10^{\circ}$ would have to be only two times larger than the corresponding stimulus at fixation. On the other hand, whether or not an $E_{2}$ value is $15^{\circ}$ or $50^{\circ}$ makes little difference in the relative scaling required at fixation versus $10^{\circ}$ eccentricity. If $E_{2}=15^{\circ}$, a stimulus at $10^{\circ}$ would have to be .67 times larger than the corresponding stimulus at fixation, and if $E_{2}=50^{\circ}$, a stimulus at $10^{\circ}$ would have to be only .2 times larger than the corresponding stimulus at fixation. The differences between these two scalings start to enter the range of sampling error of the underlying thresholds from which they are derived.

\section{GENERAL DISCUSSION}

The two data collection and fitting procedures used in this study yielded comparable results. The most notable difference was associated with sampling method. Contrasts at threshold for small stimuli were larger when data were obtained using the Poirier-Gurnsey method. A possible reason for this difference is that the Melmoth method allows the observer to selectively attend to a stimulus of one particular size and perhaps as a conse- quence, more effectively engage mechanisms optimally tuned to that stimulus. Considerable evidence indicates that spatially directed attention can modulate neural processing at the attended location (i.e., Huk \& Heeger, 2000; Kastner \& Ungerleider, 2001). With the Poirier-Gurnsey sampling method, stimuli vary in contrast and size from trial to trial. The observer may have to distribute attention across several mechanisms to perform the task, a process that may be most disruptive for the smallest stimuli. Such a disruption is not seen for large stimuli, in which the minimal contrast values at threshold were very similar across sampling methods.

Previous reports have suggested that contrast scaling may be required in some instances in order to obtain valid estimates of size scaling associated with the task. The $E_{2}$ values for contrast obtained in this study were very large (average $E_{2 \text { contrast }}=184.24^{\circ}$ and $18.5^{\circ}$ for the Poirier-Gurnsey and Melmoth procedures, respectively). Although these average values differ substantially, they are both extremely large. Thus, stimuli in the asymptotic length range (e.g., $>20^{\circ}$ ) have to be moved at least $18^{\circ}$ into the periphery before contrast at threshold must double to match foveal performance. Therefore, it is fair to say that orientation discrimination requires little to no contrast scaling to achieve excellent fits to the data.

Large $E_{2}$ values indicate that stimulus properties (e.g., size or contrast) must be increased only modestly with eccentricity in order to match foveal performance. Across all analyses described above (and in note 3 ), $E_{2 \text { size }}$ values ranged from $3.71^{\circ}$ to $6.36^{\circ}$. To determine whether contrast scaling has an important effect on $E_{2 \text { size }}$ values, we repeated all fitting procedures without scaling contrast. In other words, we carried out a standard size-scaling analysis and ignored any effects of contrast. We found that $E_{2 \text { size }}$ values averaged $5.82^{\circ}$ (range $=$ $2.83^{\circ}$ to $\left.9.93^{\circ}\right)$ with little drop in explained variance $(3 \%$ reduction on average). In other words, ignoring contrast has very little effect on explained variance and average $E_{2 \text { size }}$ values. Therefore, the large $E_{2 \text { size }}$ values found at near-threshold contrasts are not attributable to the need to scale contrast.

In fact, $E_{2 \text { size }}$ values recovered at threshold contrasts are often large whether or not contrast scaling is required. For example, Melmoth et al. (2000a) reported $E_{2 \text { size }}$ values for face discrimination ranging from $2.39^{\circ}$ to $25.7^{\circ}$ with an average across subjects of $8.81^{\circ} . E_{2 \text { contrast }}$ values ranged from $1.92^{\circ}$ to $7.51^{\circ}$ with an average of $5.53^{\circ}$. Mäkelä et al. (2001) found $E_{2 \text { size }}$ values for face detection of $2.73^{\circ}$ and $3.19^{\circ}$ for two subjects with corresponding $E_{2 \text { contrast }}$ values of $5.26^{\circ}$ and $14.5^{\circ}$. Melmoth et al. (2000b) observed that $E_{2 \text { size }}$ values of $3.60^{\circ}$ and $6.38^{\circ}$ equated performance across eccentricities for phasedistortion detection in both polar-grating and bandpassfiltered face stimuli. The authors reported that contrast scaling was not required. (We reanalyzed the data of Melmoth et al. [2000b] using a data-fitting method that solves for $E_{2 \text { size }}$ and $E_{2 \text { contrast }}$ simultaneously and found 
$E_{2 \text { size }}$ values for size ranging from $5.05^{\circ}$ to $33^{\circ}$ and $E_{2 \text { con- }}$ trast values ranging from $10.36^{\circ}$ to $694^{\circ}$.) Thus, $E_{2 \text { size }}$ values are often fairly large at near-threshold contrasts.

It could be argued that our results do not reflect the orientation selectivity of the cortex but reflect contrast sensitivity associated with precortical mechanisms. After all, some researchers have measured grating contrast sensitivity using a methodology where the forcedchoice component must distinguish between horizontal and vertical gratings, which really cannot be considered an orientation-discrimination task. Furthermore, our recovered $E_{2}$ values are in the range normally associated with grating resolution. Therefore, we have to address the claim that the orientation component of our stimulus serves only as a useful means by which a forced-choice methodology can establish contrast thresholds.

First, it is unrealistic to consider discriminating a $90^{\circ}$ difference as qualitatively similar to discriminating a $1.5^{\circ}$ difference. For example, Thomas and Gille (1979) measured detection and orientation-identification accuracy as a function of contrast. For large orientation differences, detection and identification accuracy were identical; that is, the probability of detecting the stimulus was equal to the probability of correctly identifying it. As the orientation difference between the discriminanda was reduced, identification accuracy dropped relative to detection accuracy. Clearly, small orientation differences cannot be used to measure the limits of contrast sensitivity, although large orientation differences can be. This kind of result may be understood in terms of the orientation bandwidths estimated from human psychophysical studies (Phillips \& Wilson, 1984; Thomas \& Gille, 1979) and physiological studies in the macaque (DeValois, Yund, \& Hepler, 1982). Half-amplitude orientation thresholds have been found to range from about $\pm 10^{\circ}$ or $15^{\circ}$ to $30^{\circ}$. Therefore, horizontal and vertical gratings activate populations of units with nonoverlapping sensitivities. As a result, orthogonal gratings can be used to assess contrast sensitivity of precortical mechanisms; the task can hardly be seen as challenging the ability of the cortex to resolve orientation differences. Put another way, discriminating horizontal from vertical can be seen as involving labeled lines (e.g., Watson \& Robson, 1981), whereas discriminating a $1.5^{\circ}$ orientation difference clearly does not.

Second, consider any level of contrast in the top panels of Figure 2. A $1.5^{\circ}$ orientation difference represents the orientation-discrimination threshold for that level of contrast; the orientation difference increases or decreases, accuracy will change. All data points (interpolated or otherwise) in Figure 2 having contrast $c$ indicate that stimulus size must be increased with eccentricity to maintain threshold performance. Therefore, our method and task are appropriate to assessing size scaling and orientationselective cortical mechanisms at low stimulus contrasts.

Finally, Sally and Gurnsey (2003, Experiment 1) also used a fixed orientation difference $\left( \pm 1.5^{\circ}\right)$ between two broadband stimuli and measured accuracy as a function of stimulus size. They found small $E_{2}$ values $\left(1.24^{\circ}\right.$ and $1.48^{\circ}$ for two subjects). The critical difference between Sally and Gurnsey (2003, Experiment 1) and the present experiments is the contrast of the stimuli. Sally and Gurnsey (2003, Experiment 1) used the maximum available screen contrast, whereas here, of course, we varied contrast to achieve a threshold level of accuracy. Therefore, the $E_{2 \text { size }}$ values recovered in an orientationdiscrimination task involving a fixed orientation difference depends critically on stimulus contrast, as suggested by the literature reviewed earlier.

$E_{2 \text { size }}$ values are clearly smaller when they were recovered using stimuli at contrasts well above detection threshold. This effect may arise, in part, because highcontrast methods generally use stimuli that have achieved equivalent levels of perceived contrast across eccentricities. Cannon (1985) showed that at physical (Michelson) contrasts of 0.33 and above, the perceived contrast of sine wave gratings showed essentially no change in eccentricity (to $40^{\circ}$ ), even though there was an approximately 10 fold variation in detection thresholds. Similarly, Sally and Gurnsey (2004) showed that at physical (Michelson) contrasts of .383 and above, the perceived contrasts of the stimuli used in the present study showed no change across eccentricities $\left(0^{\circ}\right.$ to $\left.15^{\circ}\right)$ and sizes $(45 \mathrm{~min}$ arc to $\left.12^{\circ}\right)$. $\left(E_{2}\right.$ values for orientation discrimination in Sally and Gurnsey [2003] were calculated for stimuli at contrasts at or above this level.) Thus, $E_{2 \text { size }}$ values at contrasts well above detection threshold appear to reflect changes in the local spatial scale in the orientationselective mechanisms and are not influenced by variations in contrast.

At near-threshold contrasts, contrast and size jointly determine sensitivity to an orientation difference. Neither method used in the present study equates stimuli for relative "distance" from contrast-detection threshold or for perceived contrast. For this reason, Sally and Gurnsey (2004) recently repeated the classic Mäkelä et al. (1993) study, using stimuli that were matched in perceptual contrast to a reference stimulus presented at fixation that was two just noticeable differences above detection threshold. Stimulus contrast had to increase systematically with eccentricity (more or less following threshold curves) to maintain equivalent perceived contrast. These "matched" contrast levels were then used to test orientation discrimination across eccentricities. $E_{2 \text { size }}$ values of $3.42^{\circ}$ and $3.50^{\circ}$ were recovered. These values, though smaller than the average $E_{2 \text { size }}$ estimate of $5.64^{\circ}$ recovered in the present study, are on average a factor of 2.3 times larger than those recovered at suprathreshold contrasts using the same subjects, stimuli, and testing conditions. These findings suggest that although methodological factors can have some impact on size scaling, the general rule seems to be that larger $E_{2 \text { size }}$ values are found at near threshold contrasts than at suprathreshold contrasts.

As mentioned at the beginning of this article, it is possible that large $E_{2 \text { size }}$ values are recovered at near-threshold contrasts because of dynamic changes in the spatial struc- 
ture of receptive fields. Recent physiological experiments in the macaque visual cortex have shown that there can be a two- to four-fold increase in VI receptive field size when measured with the same optimal stimulus at high and low contrasts (Kapadia et al., 1999; Sceniak et al., 1999). Psychophysical analogues of this effect have been provided recently. Mareschal et al. (2002) measured orientation-discrimination thresholds for sine wave gratings in the presence of surround patterns of similar orientation and spatial frequency. These surround stimuli have a detrimental effect on thresholds unless they are spatially separated from the gratings. Mareschal et al. showed that the influence of the surround on orientation thresholds was maintained over larger gap separations when both were presented at low contrast. Mareschal and Shapley (2004) have also examined the effects of contrast and size on orientation discrimination and have successfully interpreted their results in terms of the spatial reorganization of receptive fields at low-stimulus contrasts.

$E_{2 \text { size }}$ values for orientation discrimination presumably reflect changes in the local spatial scale of orientationselective mechanisms with eccentricity. That is, stimulus size must increase with eccentricity to maintain equivalentto-foveal levels of performance because of a concomitant change in the size of orientation-selective filters or receptive fields at visual cortex. If increases in receptive fields at low contrasts are relatively greater at the fovea than in the periphery, less spatial scaling (i.e., larger $E_{2 \text { size }}$ values) would be required to equate orientationdiscrimination performance. This possibility has not yet been evaluated in physiological studies.

\section{Conclusions}

We conclude that $E_{2 \text { size }}$ values recovered in an orientation-discrimination task at near-threshold contrasts are greater than those recovered at contrasts well above detection threshold. Only modest contrast scaling is required in the orientation-discrimination task, and contrast scaling has little effect on the $E_{2 \text { size }}$ values recovered. The Poirier-Gurnsey and Melmoth procedures yield similar results and lead to similar conclusions about (1) $E_{2 \text { size }}$ values recovered at near-threshold contrasts, and (2) the effect that contrast scaling has on the recovered $E_{2 \text { size }}$ values. The difference between the $E_{2 \text { size }}$ values recovered at contrasts well above detection threshold and those recovered near detection threshold may reflect contrast-dependent changes in the organization of cortical receptive fields.

\section{REFERENCES}

Barrett, B. T., Morrill, P., \& Whitaker, D. (2000). Compound grating discrimination in extrafoveal and amblyopic vision. Experimental Brain Research, 131, 225-235.

Bennett, P. J., \& Banks, M. S. (1991). The effect of contrast, spatial scale, and orientation on foveal and peripheral phase discrimination. Vision Research, 31, 1759-1786.

Brainard, D. H. (1997). The Psychophysics Toolbox. Spatial Vision, 10, 443-446.
Cannon, M. W., JR. (1985). Perceived contrast in the fovea and periphery. Journal of the Optical Society of America A, 2, 1760-1768.

DeValois, R. L., Yund, E. W., \& HePler, N. (1982). The orientation and direction selectivity of cells in macaque visual cortex. Vision Research, 22, 531-544.

Farrell, J. E., \& Desmarais, M. (1990). Equating character-identification performance across the visual field. Journal of the Optical Society of America A, 7, 152-159.

Higgins, K. E., Arditi, A., \& KNoblauch, K. (1996). Detection and identification of mirror-image pairs in central and peripheral vision. Vision Research, 36, 331-337.

Huk, C. A., \& Heeger, D. J. (2000). Task-related modulation of visual cortex. Journal of Neurophysiology, 83, 3525-3536.

JoHNSTON, A. (1987). Spatial scaling of central and peripheral contrast sensitivity functions. Journal of the Optical Society of America A, 4, 1583-1593.

Johnston, A., \& Wright, M. J. (1986). Matching velocity in central and peripheral vision. Vision Research, 26, 1099-1109.

Kapadia, M. K., Westheimer, G., \& Gilbert, C. D. (1999). Dynamics of spatial summation in primary visual cortex of alert monkeys. Proceedings of the National Academy of Sciences, 96, 12073-12078.

Kastner, S., \& Ungerleider, L. G. (2001). The neural basis of biased competition in human visual cortex. Neuropsychologia, 39, 1263-1276.

Klein, S. A., \& Levi, D. M. (1987). Position sense of the peripheral retina. Journal of the Optical Society of America A, 4, 1543-1553.

Koenderink, J. J., Bouman, M. A., Bueno de Mesquita, A. E., \& SLAPPENDEL, S. (1978). Perimetry of contrast detection thresholds of moving spatial sine wave patterns: III. The target extent as a sensitivity controlling parameter. Journal of the Optical Society of America, 68, 854-860.

Levi, D. M., Klein, S. A., \& Aitsebaomo, A. P. (1984). Detection and discrimination of the direction of motion in central and peripheral vision of normal and amblyopic observers. Vision Research, 24, 789-800.

Levi, D. M., Klein, S. A., \& Aitsebaomo, A. P. (1985). Vernier acuity, crowding and cortical magnification. Vision Research, 25, 963-977.

MäKelä, P., NÄsänen, R., Rovamo, J., \& Melmoth, D. (2001). Identification of facial images in peripheral vision. Vision Research, 41, 599-610.

MäKelä, P., WhitaKer, D., \& Rovamo, J. (1993). Modelling of orientation discrimination across the visual field. Vision Research, 33, 723-730.

Mareschal, I., Henrie, J. A., \& Shapley, R. M. (2002). A psychophysical correlate of contrast-dependent changes in receptive field size. Vision Research, 42, 1879-1887.

Mareschal, I., \& Shapley, R. M. (2004). Effects of contrast and size on orientation discrimination. Vision Research, 44, 57-67.

Melmoth, D. R., Kukkonen, H. T., MäKelë, P., \& Rovamo, J. M. (2000a). The effect of contrast- and size-scaling on face perception in foveal and extrafoveal vision. Investigative Ophthalmology \& Visual Science, 41, 2811-2819.

Melmoth, D. R., Kukkonen, H. T., Mäkelä, P., \& Rovamo, J. M. (2000b). Scaling extrafoveal detection of distortion in a face and grating. Perception, 29, 1117-1126.

Melmoth, D. R., \& Rovamo, J. M. (2003). Scaling of letter size and contrast equalises perception across eccentricities and set sizes. $\mathrm{Vi}$ sion Research, 43, 769-777.

PelLI, D. G. (1987). The ideal psychometric procedure [Abstract]. Investigative Ophthalmology \& Visual Science, 28 (Suppl.), 366.

PelLI, D. G. (1997). The VideoToolbox software for visual psychophysics: Transforming numbers into movies. Spatial Vision, 10, 437-442.

Pelli, D. G., \& Zhang, L. (1991). Accurate control of contrast on microcomputer displays. Vision Research, 31, 1337-1350.

Phillips, G. C., \& Wilson, H. R. (1984). Orientation bandwidths of spatial mechanisms measured by masking. Journal of the Optical Society of America A, 1, 226-232.

PoIRIER, F. J. A. M., \& GURNSEY, R. (2002). Two eccentricity-dependent limitations on subjective contour discrimination. Vision Research, 42, 227-238.

Rovamo, J., \& Virsu, V. (1979). An estimation and application of the 
human cortical magnification factor. Experimental Brain Research, 37, 495-510.

Rovamo, J., VIRSU, V., \& NÄSÄNEN, R. (1978). Cortical magnification factor predicts the photopic contrast sensitivity of peripheral vision. Nature, 271, 54-56.

SaARINEn, J., Rovamo, J., \& Virsu, V. (1989). Analysis of spatial structure in eccentric vision. Investigative Ophthalmology \& Visual Science, 30, 293-296.

SAlly, S. L., \& GURNSEY, R. (2003). Orientation discrimination in foveal and extra-foveal vision: Effects of stimulus bandwidth and contrast. Vision Research, 43, 1375-1385.

SALLY, S. L., \& GURNSEY, R. (2004). Orientation discrimination across the visual field: Size scaling estimates at near-threshold levels of contrast. Vision Research, 44, 2719-2727.

Sceniak, M. P., Hawken, M. J., \& Shapley, R. M. (2002). Contrastdependent changes in spatial frequency tuning of macaque V1 neurons: Effects of a changing receptive field size. Journal of Neurophysiology, 88, 1363-1373.

Sceniak, M. P., Ringach, D. L., Hawken, M. J., \& Shapley, R. M. (1999). Contrast's effect on spatial summation by macaque V1 neurons. Nature Neuroscience, 2, 733-739.

SERWAY, R. A. (1992). Physics for scientists and engineers with modern physics (3rd ed.). Fort Worth: Saunders College Publishing.

Strasburger, H., Harvey, L. O., JR., \& Rentschler, I. (1991). Contrast thresholds for identification of numeric characters in direct and eccentric view. Perception \& Psychophysics, 49, 495-508.

Strasburger, H., Rentschler, I., \& Harvey, L. O., JR. (1994). Cortical magnification theory fails to predict visual recognition. European Journal of Neuroscience, 6, 1583-1588.

Thomas, J. P., \& Gille, J. (1979). Bandwidths of orientation channels in human vision. Journal of the Optical Society of America, 69, 652-660.

WATSON, A. B. (1987). Estimation of local spatial scale. Journal of the Optical Society of America A, 4, 1579-1582.

Watson, A. B., \& Pelli, D. G. (1983). QUEST: A Bayesian adaptive psychometric method. Perception \& Psychophysics, 33, 113-120.

WATSON A. B., \& RoBson J. G. (1981). Discrimination at threshold: Labelled detectors in human vision. Vision Research, 21, 1115-1122.

WESTHEIMER, G. (1982). The spatial grain of the perifoveal visual field. Vision Research, 22, 157-162.

Weymouth, F. W. (1958). Visual sensory units and the minimal angle of resolution. American Journal of Ophthalmology, 46, 102-113.

Whitaker, D., Latham, K., MäKelä, P., \& Rovamo, J. (1993). Detection and discrimination of curvature in foveal and peripheral vision. Vision Research, 33, 2215-2224.
Whitaker, D., Rovamo, J., MacVeigh, D., \& Mäkelë, P. (1992). Spatial scaling of vernier acuity tasks. Vision Research, 32, 1481-1491.

WRIGHT, M. J. (1987). Spatiotemporal properties of grating motion detection in the center and periphery of the visual field. Journal of the Optical Society of America A, 4, 1627-1633.

\section{NOTES}

1. There is some controversy concerning this point. Bennett and Banks (1991) found that a certain type of phase discrimination $\left(90^{\circ} \mathrm{vs}\right.$ $270^{\circ}$ shift of second harmonic of a compound grating, relative to the fundamental) could not be equated through simple spatial scaling. Failure of size scaling in a simple phase-discrimination task might appear to contradict the position of Melmoth et al. (2000b). Recently, however, Barrett, Morrill, and Whitaker (2000) found that $E_{2}$ values ranging from $.35^{\circ}$ to $.69^{\circ}$ were sufficient to eliminate the eccentricity dependence in discrimination accuracy data for the $90 / 270$ phase shifts; that is, 90/270 phase shifts can be size scaled.

2. Previous work has fixed contrast and varied size and orientation differences (e.g., Mäkelä et al., 1993), whereas we have fixed the orientation difference and varied size and contrast. It is possible to compute 3-D response surfaces (performance as a function of size, contrast, and orientation difference), but it is difficult as a practical matter to recover such a large data set. We therefore addressed the more focused question of the joint contributions of size and contrast to orientation discrimination, because there is a base of empirical literature (e.g., Melmoth et al., 2000a, 2000b; Melmoth \& Rovamo, 2003) against which the results can be compared.

3. We subjected all data recovered from each sampling procedure to the fitting procedure associated with the alternate method and noted relatively little change in $E_{2}$ values. For example, the $E_{2 \text { size }}$ values for data collected with the Melmoth sampling method and scaled with the Poirier-Gurnsey method were $3.71^{\circ}$ and $4.91^{\circ}$ for S.S. and S.M., respectively. Corresponding $E_{2 \text { contrast }}$ values for the two subjects were $36.90^{\circ}$ and $11.01^{\circ}\left(r^{2}\right.$ values were .96 for both subjects $)$. Conversely, the $E_{2 \text { size }}$ values for data collected with the Poirier-Gurnsey sampling method and scaled with the Melmoth sampling method were $5.94^{\circ}$ and $6.36^{\circ}$ for SS and SM, respectively. Corresponding $E_{2 \text { contrast }}$ values for the two subjects were $203^{\circ}$ and $27.6^{\circ}$ ( $r^{2}$ values were .97 for both subjects)

(Manuscript received December 4, 2003; revision accepted for publication August 13, 2004.) 